\title{
Les remédiations fantasmatiques de la violence
}

\author{
Richard Bégin \\ Université de Montréal
}

Toute émeute est un mouvement qui, par nature, est violent. Une violence qui émeut par sa mouvance même. Ce qui convient a priori au sens étymologique du terme, «émeute » provenant de esmuete, qui veut dire " mouvement, émoi ». Ensuite de quoi, suivant son sens commun (et politique), l'émeute se veut la généralisation populaire d'un mouvement pulsionnel de destruction massive qui, remarquablement, ne vise pas toujours le but ou l'intention qu'on lui prête d'emblée ou qu'on aimerait seulement lui attribuer tant le mouvant et l'instabilité sociale 
inquiètent. En effet, qu'il s'agisse d'émeutes nées, croit-on, de la frustration partisane d'assister à la défaite d'une équipe de sport professionnelle, ou bien qu'il s'agisse d'une casse collective en réaction, croit-on, à la mort d'un citoyen victime de brutalités policières, toujours l'émeute surprend en ceci qu'elle échappe à la raison qu'on tente, tant bien que mal, de lui attribuer. Plutôt mal que bien si on en juge par les nombreux débats et dialogues de sourds qui ont cours sur les tribunes publiques créées en réponse à de tels événements.

C'est que la violence d'une émeute en acte échappe inévitablement au discours qui tenterait de l'arraisonner, de lui trouver ses raisons. Rien ne permet en effet d'interpréter de manière globale un mouvement de colère qui, parce qu'il est un mouvement justement, ne cesse de varier et de se modifier, allant parfois même jusqu'à " choisir » pour cibles privilégiées les possessions matérielles de celles et ceux qui en sont les instigateurs. Combien de fois, en ce sens, s'est-on étonné de constater qu'une manifestation de la classe populaire la plus défavorisée d'un quartier débouche sur la destruction de biens et immeubles appartenant à cette même communauté? Ou bien qu'une émeute en réaction à de dramatiques inégalités sociales sombre dans le chaos le plus violent dès qu'y participe, encapuchonné, un groupe de jeunes provenant de familles aisées, voire nanties?

On comprend alors pourquoi nombre d'analystes, de journalistes et de "spécialistes d'office » s'évertuent à chercher une « raison d'être » à un mouvement collectif qui, en soi, n'en a peut-être pas, si ce n'est celle, ironiquement, de se faire violence commune. C'est cette violence commune réfléchissante qui échappe au discours en ne s'expliquant pas autrement que par 
une pulsion autodestructrice délivrée de toute distanciation critique que pourrait opérer l'émeutier qui en est habité. En fait, seule la justice semble être en mesure d'offrir un semblant d'explication - policière, au sens où l'entend Jacques Rancière — à ce phénomène.

Ne cherchons toutefois pas ici à prétendre de manière précipitée que toute émeute, parce qu'elle relève d'une violence commune réfléchissante, procure les mêmes sentiments à celles et ceux qui participent de sa formation qu'à celles et ceux qui n'en sont que les témoins plus ou moins éloignés. Il n'en demeure pas moins, cependant, que son mouvement, en effet, émeut de manière généralisée, globalisante et, si on peut se permettre ce néologisme d'actualité, blogalisante. Car il ne faut pas sous-estimer le fait que, de manière générale, le mouvement de l'émeute, où qu'elle se produise, s'étend désormais aux réseaux sociaux, aux blogues, véritables véhicules émotifs «hypermodernes » dont on ne peut négliger aujourd'hui l'importance. L'émotion du phénomène se trouve ainsi partagée quasi instantanément entre l'émeutier, le témoin et l'internaute, dans l'exacte mesure où le mouvement de l'émeute parvient à faire image, puis à circuler et à s' " écumer », dirait Peter Sloterdijk, au travers tel ou tel appareil de diffusion.

Les images prises par les «téléphones intelligents » sur les lieux d'une émeute inaugurent en cela, et de manière remarquable, ce partage blogalisé que l'on pourrait qualifier de « pulsionnel »; une pulsion produite par un mouvement en acte n'étant plus représenté par les grands médias institutionnalisés, mais bien imagé dans le registre fantasmatique de cette nouvelle forme d'immédiateté sociale qu'est l'association en réseau. Cette association n'explique rien de l'événement actuel, 
c'est un fait, mais elle n'en permet pas moins à l'émeute d'étendre virtuellement son mouvement hors du lieu physique où elle se produit actuellement. C'est ce même mouvement hors de soi - ou cette forme d'extase de la violence médiatique que tente également de maintenir les discours judiciaire et policier en traduisant devant les tribunaux ces blogueurs qui, à distance, incitent, littéralement, à poursuivre le mouvement.

\section{De la virtualisation de la violence...}

Ces images d'émeutes qui circulent et que l'on juge «immédiates» et «citoyennes», puisque dénuées d'intermédiaires institutionnels, ont un rôle médiatique sournois, non manifeste; alors qu'on aime à croire qu'elles ne font que montrer, sans intermédiaire, le mouvement actuel du phénomène, elles introduisent l'émeute dans un régime esthétique qui ne fait voir de celle-ci qu'une forme extatique d'immédiateté. D'abord, certes, du seul fait que ces images particulières prises "à chaud » sous-tendent un acte énonciatif, donc, une énonciation, un énoncé et un énonciateur, voire une prise de position; puis ensuite, du seul fait qu'elles inscrivent la violence actuelle du mouvement au sein d'un registre associatif qui, malgré l'absence d'intermédiaire institutionnel nous communiquant une représentation discursive du phénomène en acte - par le biais du « reporter » par exemple —, impose à ce même phénomène une réinscription iconique, spatiale et temporelle, hors contexte, hors de lui-même.

C'est cette réinscription extatique du phénomène qu'inaugurent les réseaux sociaux et les différents blogues en 
ceci qu'ils reconfigurent de manière subtile le mouvement actuel de l'émeute, suivant cependant un régime autre que celui de la simple représentation discursive; ils le reconfigurent suivant un régime esthétique immersif qui intègre dans sa logique l'internaute du seul fait que les images lui sont « personnellement » adressées, chez lui, sur lui, dans son écran. Un régime esthétique immersif qui implique que l'internaute reconnaisse ainsi au phénomène partagé une cause à laquelle il puisse dès lors adhérer, des victimes au travers lesquelles il peut se reconnaître et des bourreaux qui peuvent également être les siens. Il s'agit d'un registre iconique qui demeure en partie discursif du moment où l'image entraîne son spectateur - qui n'en est plus vraiment un - à croire en une simple monstration de l'actuel là où il $\mathrm{y}$ a avant tout communication, médiation et, surtout, virtualisation du montré.

Dans Qu'est-ce que le virtuel ?, Pierre Lévy nous rappelle que le processus de virtualisation, loin de produire de l'illusoire et du simulacre, réfère à la création et, surtout, à la problématisation de l'actuel. Ce qui s'oppose au processus d'actualisation, lequel concerne pour sa part la solution à un problème créé. La virtualisation est une problématisation en ce qu'elle questionne le phénomène actuel afin de le mettre en mouvement, soit, littéralement, de l'introduire dans le registre de l'émotion. Le réseau permettant la circulation d'images captées sur le vif inscrit donc celles-ci dans un processus s'appropriant le mouvement actuel de l'émeute; un processus qui médiatise l'émotion «à chaud » et qui a pour conséquence d'en créer de nouvelles, pouvant, elles, être partagées. C'est au moment de cette appropriation que s'évanouit le mouvement actuel de l'émeute, au moment exact, bref, où surgit une émotion virtuelle, partagée, extatique, qui, puisqu'elle ne cesse 
de circuler et d'être remédiée par les nombreux appareils de diffusion, ne cesse également de redéfinir la figure même de l'émeute.

L'affaire du «matricule 728 » est exemplaire de ce processus de virtualisation. Lors des manifestations étudiantes du printemps 2012, une agente du Service de police de la Ville de Montréal aspergea quelques manifestants d'une généreuse dose de poivre de Cayenne. Cet événement anecdotique a été capté par le téléphone portable d'un témoin, et l'image de celuici circula au sein des réseaux sociaux, des sites de partage vidéo et reprise en boucle aux informations télévisées du soir. La circulation de ces images a permis la production d'un mythe, le «matricule $728 »$, qui ne fait finalement que prolonger l'imaginaire de la brutalité policière. L'actualité même de l'anecdote fut ainsi problématisée par une circulation iconique qui aura tôt fait de traduire le mouvement actuel de la manifestation en une émotion socialement, culturellement et politiquement tendancieuse. C'est cette émotion virtuelle qui, en retour, nous entraîne à lire le mouvement en acte par la lorgnette du fantasme. Aussi «matricule 728 » sera-t-elle ensuite victime de son propre mythe au moment où, lors d'une intervention policière à l'automne 2012 cette fois, sa brutalité sera de nouveau mise en cause. Une arrestation musclée effectuée par cette même agente sera captée par le téléphone portable d'un témoin et servira de preuve afin, éventuellement, de porter des accusations contre cette dernière. Ces accusations réfèreront ainsi à des faits technologiquement orientés et aux agissements d'une agente qui, bien malgré elle, incarne désormais la figure fantasmatique de la brutalité policière. 
Ce qui se perd dans le partage extatique de l'émotion, l'acte comme tel, se gagne ainsi en flux fantasmatique. Comme si la circulation des images avaient permis la production ex nihilo d'un nouvel événement, issu, celui-là, d'un appareillage technique inaugurant à sa manière une perception inédite des choses, bref, une esthétique. Les Exercices spirituels de SaintIgnace de Loyola ne sont pas bien loin. Car dès l'inscription médiatique - ou la remédiation - du mouvement actuel et singulier de l'émeute, apparaît l'interface de virtualisation, soit, justement, l'appareil qui, à la manière de l'appareil spirituel que dissimule l'œuvre religieuse, dessine les formes de ce mouvement afin d'en traduire les «véritables» apparences. Tout appareil sert avant tout à donner ses apparences à l'apparaître. C'est ainsi que l'apparence et l'appareil ne font qu'un, l'appareil médiatique de diffusion et l'appareil spirituel chrétien se partageant ainsi le privilège de l'émotion esthétique. Il serait pourtant faux de croire que le mouvement actuel de l'émeute, camouflé dès lors derrière le voile de l'interface, ne suscite plus d'émotions. Or, ce n'est plus le mouvement en acte qui émeut ici, mais l'appareil qui met cet acte en mouvement et, par conséquent, le virtualise, qui suscite l'émotion et, par voie de conséquence, nous permet de l'envisager, de lui donner un visage, une figure.

C'est ce design virtuel de l'émotion que prolonge sans le savoir l'utilisateur du dispositif portable en intervenant dans le mouvement actuel d'une émeute, d'une intervention policière ou d'une manifestation, par exemple, afin d'en prélever les germes d'une apparence qui ne sera telle qu'au moment du partage, de la communication et de la remédiation. Or cet inconscient de l'utilisateur n'en est pas vraiment un du moment où l'on considère qu'un tel type de témoignage visuel permet de 
produire un événement distinct, technologiquement orienté celui-là. Cette événementialité seconde naît alors d'un dispositif technique qui, comme tout dispositif, nous rappelle Agamben, a la capacité d'orienter et de déterminer les conduites du sujet: «Les dispositifs doivent toujours impliquer un processus de subjectivation. » (p. 27) Ce pourquoi on ne peut faire l'impasse sur la nature inédite du témoin qu'instaure une telle virtualisation des phénomènes en acte. Cette virtualisation de l'actuel a pour ainsi dire la faculté de créer à la fois l'événement, ses témoins, ses bourreaux et ses victimes.

On comprend ici qu'on ne peut faire adéquatement l'économie du partage des images sans y associer l'appareillage virtuel - et non plus seulement l'intermédiaire institutionnel - sans qui cette apparence sociale, culturelle et politique de l'émeute ne saurait exister. C'est donc dire qu'à l'émeute et à son mouvement actuel ainsi médiatisés ne correspond plus que son écriture, son apparat, bref, que ce qui en dessine pour nous l'esthétique. En somme, à l'émeute ainsi mise en mouvement ne correspond plus que son design émotif. Le médium, c'est le message, certes, mais on oublie trop souvent que le médium, c'est aussi le fantasme. Il s'agit bien d'un fantasme dans la mesure où toute apparence relève du design, lequel participe de la virtualisation même du mouvement actuel, de sa figuration. La violence d'une émeute ainsi appareillée ne se réduit évidemment pas forcément à l'illusion que procurerait, par exemple, un rêve éveillé, mais il nous faut néanmoins reconnaître dans la mise en mouvement de l'acte l'intervention de l'écran qui n'est autre que le tiers acteur intervenant entre celui qui y était actuellement et celui qui, actuellement, regarde; un acteur en ce qu'il ne fait pas que transmettre de l'un à l'autre, mais en ce qu'il opère une véritable traduction de l'événement 
au sens où l'entend Bruno Latour dans sa théorie de l'acteurréseau, une traduction ou une virtualisation qui est toujours susceptible de satisfaire l'imaginaire de l'un comme de l'autre.

\section{...à son développement fantasmatique}

Il y a de nos jours, à ne pas en douter, une véritable culture de l'écran qui jette sur tout événement ou mouvement actuel l'éventualité - devenue nécessaire, croit-on - de sa propre traduction médiatique, voire de sa propre virtualisation. Ce qui ne va pas sans causer quelques problèmes éthiques du moment où au passage à l'écran du phénomène actuel correspond son esthétisation, l'écran interjetant de fait une surface d'inscription sensuelle entre les deux faces de l'actuel, ici et làbas. C'est cette traduction esthétique qui, parce qu'elle parachève en outre le régime immersif de l'association en réseau, qui redessine les formes mêmes de la violence jusqu'à ne nous en faire voir que ses remédiations fantasmatiques. Il nous faut alors bien comprendre le lien entre cette traduction de l'événement actuel et la propagation fantasmatique de celuici si l'on souhaite saisir en quoi il existerait aujourd'hui, pourrait-on dire, une figuration inédite de la violence.

Prenons l'exemple du film Frontière(s) de Xavier Gens, qui représente un cas remarquable de figuration en ce qu'il fait clairement le lien «fantasmatique» entre le mouvement médiatisé et « traduit » de l'émeute et l'incorporation extatique de son actualité au sein d'un imaginaire narratif, ici pour le moins grotesque. Le film débute avec des images ayant vraisemblablement pour origine les enregistrements d'une 
véritable émeute. Cette succession d'images actuelles, tournées à l'aide de téléphones portables ou de tout autre dispositif numérique mobile, compose à elle seule un phénomène violent par la juxtaposition des prises de vue qui, chacune, offre une traduction singulière de l'événement et le virtualise par la même occasion. Aussi ces images médiatisent-elles le mouvement actuel de l'événement et en instaurent-elles déjà, pour nous, l'esthétique.

Ce que l'écran mobile et singulier "projette » ainsi déjà sur le mouvement actuel de l'événement, jusqu'à le couvrir entièrement, c'est l'expression de sa médiumnité, soit, si l'on veut, sa faculté désormais innée d'être communiqué et de se voir aussitôt approprié par autrui, où qu'il soit, qui qu'il soit. L'écran fait ainsi "écran", si l'on peut dire, à l'actualité du phénomène tout en en instituant les formes émotionnelles dans une écumisation généralisée ou une remédiation blogalisée de laquelle il puisera désormais toute sa réalité et ses «véritables » apparences, ce pourquoi il semble de bon ton de considérer «authentiques» les images circulant dans les réseaux sociaux alors même qu'il est de bon ton de douter de la pleine objectivité de celles diffusées via les intermédiaires institutionnels. Or on oublie que cette « démocratisation » de la représentation entraîne avec elle une désinstitutionnalisation des perspectives qui a pour conséquence de produire un "synoptisme» imaginaire, à défaut de parachever le panoptisme symbolique auquel les intermédiaires institutionnels nous avaient habitués.

Qu'est-ce à dire? Revenons au film de Xavier Gens, qui nous offre un bel exemple de ce qui advient des images du moment où leur sens même est livré à la remédiation et à la 
traduction. Après quelques minutes d'un montage d'images de "véritables » émeutes, le récit nous entraîne dans la vie tumultueuse d'une bande de jeunes voyous aux prises avec les autorités et cherchant à tout prix à fuir une ville livrée au saccage et au chaos. S'ensuit alors une aventure grotesque, violente, au cours de laquelle les jeunes en question seront confrontés, dans une campagne reculée, à une famille néo-nazie qui ne cherche qu'à démembrer un à un les étrangers, citadins de préférence, de passage dans son auberge. Bien des critiques et cinéphiles se seront interrogés, avec raison, sur le sens à donner aux premières images du film dans la mesure où plus jamais le récit ne fera mention de ces émeutes, aussi «véritables » soient-elles, ni de leur effet, ni de leur cause. On nous fait seulement comprendre au tout début du récit qu'un parti politique d'extrême-droite a pris le pouvoir en France et que les jeunes des banlieues souffrent d'une vague violente de brutalité policière.

Le lien entre les émeutes des banlieues parisiennes en 2005 et la présence du Front National au second tour des élections françaises de 2002 est évident. Mais la relation entre la violence actuelle des émeutes urbaines et les violences imaginaires commises par les paysans néo-nazis l'est beaucoup moins. C'est ici qu'on ne peut manquer de prendre en compte la fantasmatisation d'un événement en acte - l'émeute - dont le récit filmique ne garde, au final, qu'une émotion virtuelle, figurale, n'ayant strictement rien à voir avec le phénomène actuel dont elle tire pourtant son origine. C'est le fantasme de la violence médiatisée qui en vient à prendre lieu et place de l'actuel et, par conséquent, à instaurer, une esthétique de la violence qui n'a plus de cause que sa propre traduction. La violence du film en vient par le fait même à posséder sa réalité 
et à exprimer une émotion délivrée de toute origine sociale ou politique qui en expliciterait la cause. On peut comprendre alors qu'une émeute ainsi traduite puisse devenir fantasmatique en ce que ses "véritables » apparences s'avèrent être davantage l'œuvre d'une circulation d'images que d'une explicitation symbolique.

$\mathrm{Du}$ panoptisme symbolique qu'opère l'intermédiaire institutionnel en ne faisant voir du phénomène que ce qui doit être montré, au synoptisme généralisé qu'instaure la circulation des images, deux voies d'accès à l'actuel s'affrontent: l'explicitation, d'une part, et la virtualisation, de l'autre. Mais dans les deux cas, l'accès se fait au prix du phénomène. Dans son article «The Viewer Society: Michel Foucault's 'Panopticon' revisited », Thomas Mathiesen analyse les réciprocités existant entre la société de surveillance décrite par Foucault à travers l'idée benthamienne du Panopticon et la société du spectacle que Mathiesen définit au moyen du concept de Synopticon. Si Foucault décrit une logique sociétale moderne dans laquelle une minorité maîtrisant les différents mécanismes panoptiques de surveillance détient de facto sur la majorité un pouvoir entendu, Mathiesen, pour sa part, évoque une logique sociétale contemporaine qui voit, au contraire, le pouvoir basculer du côté de la majorité en raison des différents appareils médiatiques permettant à l'ensemble (syn) de voir (opticon), d'enregistrer et de diffuser les images d'une minorité politique, juridique ou institutionnelle. Mathiesen voit dans le processus $\mathrm{du}$ sondage d'opinions le symptôme de ce basculement synoptique en ce que ce processus d'échantillonnage évoque une logique sociétale fondée sur le contrôle de l'image par le public. Cette logique, héritée du phénomène télévisuel, concerne ainsi, toujours selon Mathiesen, une culture de 
« voyants », d'usagers et d'utilisateurs qui non seulement ont la capacité d'énoncer ce qu'ils désirent voir au moment et à la fréquence qui leur convient, mais, apparemment, possèdent en outre le pouvoir de choisir et de produire les images qu'ils consomment. Or, comme le soutient Mathiesen, ce pouvoir individuel est une illusion. Les modalités synoptiques n'excluent pas les modalités panoptiques; bien au contraire; elles se renforcent réciproquement. Il n'est qu'à penser aux résultats d'un sondage d'opinions, lesquels s'inscrivent ipso facto au sein d'une économie marchande et technologique qui «voit» dans chaque individu un consommateur ou un usager potentiel.

Publié en 1997, le texte de Mathiesen a connu une certaine fortune critique, la modalité synoptique ayant été reprise, entre autres, par Zygmunt Bauman en 1999 dans Le coût humain de la mondialisation afin de définir une société mondialisée caractérisée par la mobilité des sujets. Mais un retour à ce concept ainsi qu'aux différentes déclinaisons technologiques qu'il suppose est aujourd'hui plus que nécessaire étant donné l'explosion des technologies numériques «mobiles » et la popularité grandissante ainsi que la propagation exponentielle des «téléphones intelligents » de troisième et de quatrième génération ( $3 \mathrm{G}$ et $4 \mathrm{G}$ ). Comme on le sait, les appareils numériques mobiles participent de la définition d'une culture occidentale de plus en plus orientée vers la désinstitutionnalisation de la création, la numérisation de la représentation, la délocalisation de la communication, bref, vers un synoptisme surdimensionné. Aussi cette "définition », qui est en partie celle de la culture numérique actuelle, invite-t-elle à la réflexion puisqu'elle suppose de nouvelles manières d'être ensemble, de s'associer, ainsi que 
d'inédites particularités perceptives. Ce sont ces particularités perceptives de l'appareil numérique mobile qu'il nous faut prendre en compte dans l'instauration d'une remédiation fantasmatique de la violence qui, parce qu'elle suppose une économie du partage et de la circulation, contribue à la virtualisation de celle-ci, là même où les particularités perceptives de l'appareil institutionnel contribuaient hier encore à sa symbolisation.

Ce à quoi un film comme Frontière(s) de Xavier Gens nous invite alors à réfléchir, c'est à l'expression de cette économie. Car, plus qu'un récit grotesque, le film de Gens se veut, peut-être sans le savoir, le développement fantasmatique d'un phénomène actuel - l'émeute -, dont l'émotion n'a plus de sens qu'en fonction de sa propre traduction médiatique que lui impose le synoptisme engrangé par la culture numérique actuelle. Aussi, en nous interrogeant sur ce que les écrans et la circulation des images font à l'événement en acte, nous interrogeons-nous du même coup sur la nature même de la violence contemporaine et sur les manières d'en faire le récit. Car ce qui fait ici violence dans le film de Gens, c'est moins le monstre tapi dans la campagne reculée guettant sa proie que la projection d'un mouvement urbain, actuel et concret, à l'extérieur de lui-même. Le paysan néo-nazi ne devenant de la sorte que l'incarnation extatique, figurale, d'une violence urbaine non seulement délivrée de son contexte, hors d'elle-même, mais livrée à ces «véritables» apparences auxquelles se résume sa 
virtualisation. De même, le film de Gens ainsi que l'affaire «matricule 728 » posent-ils bien cette question éminemment pertinente : où est donc passée l'émeute? Le film y répond à sa façon : ailleurs, c'est-à-dire en nous, avec nous, sur nos écrans fantasmatiques. La violence «virtuelle» n'est donc pas si étrangère à la violence " actuelle » de l'émeute en ceci qu'elles sont, toute deux, livrées, en dehors de tout discours institutionnel, au seul partage pulsionnel autodestructeur qui circule d'un spectateur à l'autre, de l'imaginaire du producteur à celui de son récepteur.

\section{Bibliographie}

AGAMBEn, Giorgio. (2007), Qu'est-ce qu'un dispositif ? Paris, Payot.

BAUMAN, Zygmunt. (1999), Le coût humain de la mondialisation, Paris, Hachette.

DE LoyolA, Ignace. (1991), Exercices spirituels, Paris, Arléa.

LATOUR, Bruno. (2007), Changer de société, refaire de la sociologie, Paris, La Découverte.

LÉvY, Pierre. (1998), Qu'est-ce que le virtuel ? Paris, La Découverte.

MATHIESEN Thomas. (1997), «The Viewer Society. Michel Foucault's 'Panopticon' Revisited », Theoretical Criminology, vol. $1, \mathrm{n}$ - 2, p. 215-234.

RANCIÈRE, Jacques. (2002), La mésentente: politique et philosophie, Paris, Galilée.

SloterdijK, Peter. (2002), Bulles. Sphère I, Paris, J.-J. Pauvert. 


\title{
Résumé
}

Filmer un événement violent à l'aide d'un appareil numérique mobile pour ensuite le diffuser sur Internet contribue à modifier le sens même de cet événement. Du sens réel de l'événement ne reste plus alors qu'une image "remédiée » de sa violence qui peut être traduite à son tour au gré des différentes associations médiatiques virtuelles qui n'en gardent bien souvent que l'aura fantasmatique, qu'elle soit narrative, politique ou idéologique. Cette remédiation fantasmatique de la violence qu'inaugurent les appareils mobiles se doit d'être analysée afin que l'on puisse en dégager les nouvelles figurations de la violence symptomatiques de la culture numérique actuelle. Nous analyserons cette remédiation en établissant un lien entre les images de l'émeute et l'imaginaire qu'en retient un film d'horreur comme Frontière(s) de Xavier Gens.

\begin{abstract}
Filming a violent event with a digital camera or a mobile and then broadcast it on the Internet is helping to change the very meaning of this event. The real meaning of the event that remains is a picture of a translated violence that often keeps the fantasy in a narrative, political or ideological way. This violence fantasized introduces by mobile devices must be analyzed so that we can identify new representations of violence symptomatic of the current digital culture. We will analyze this remediation by establishing a link between images of the riot and imagination in a horror movie like Xavier Gens' Frontière(s).
\end{abstract}

